\title{
Labyrinthe
}

$40 \mid 2013$

Comme les abeilles

\section{Les Foucault(s) du christianisme}

Note critique autour de Michel Foucault et le christianisme de Philippe Chevallier

\section{Julien Cavagnis}

\section{(2) OpenEdition}

Journals

Édition électronique

URL : http://journals.openedition.org/labyrinthe/4333

DOI : $10.4000 /$ labyrinthe.4333

ISSN : 1950-6031

Éditeur

Hermann

Édition imprimée

Date de publication : 1 mars 2013

Pagination : 143-151

ISBN : 9782705688400

Référence électronique

Julien Cavagnis, «Les Foucault(s) du christianisme », Labyrinthe [En ligne], 40 | 2013, mis en ligne le 01 mars 2015, consulté le 01 mai 2019. URL : http://journals.openedition.org/labyrinthe/4333 ; DOI : $10.4000 /$ labyrinthe.4333 


\section{Les Foucault(s) du christianisme}

Note critique autour de Michel Foucault et le christianisme de Philippe Chevallier

Julien CAVAGNIS

cavagnisjulien@gmail.com

Le livre de Philippe Chevallier, Michel Foucault et le christianisme, qui vient de paraitre aux ENS Éditions ${ }^{1}$, est digne d'intérêt à plusieurs titres. Voici en effet la première étude française portant sur la question du christianisme, mais aussi sur la religion, chez Michel Foucault. Il faut dire que l'Hexagone avait pris, en ce domaine, un certain retard. Il suffit d'évoquer quelques travaux anglo-saxons pour s'en rendre compte: Foucault and religion. Spiritual Corporality and Political Spirituality $^{2}$ en 2000, Michel Foucault and Theology. The Politics of Religious Experience ${ }^{3}$ en 2004, Foucault and Theology ${ }^{4}$ en 2011, pour ne citer que les plus importants ${ }^{5}$. C'est donc une certaine mise à jour de la recherche française en un domaine jusqu'à aujourd'hui délaissé qu'il faut saluer avant tout.

Nulle référence, pourtant, dans Michel Foucault et le christianisme, à un tel corpus. Philippe Chevallier adopte une position « littéraliste »,

\footnotetext{
1. Philippe Chevallier, Michel Foucault et le christianisme, Lyon, ENS Editions, coll. « La croisée des chemins », 2012.

2. Jeremy R. Carrette, Foucault and Religion. Spiritual Corporality and Political Spirituality, Londres, Routledge, 2000.

3. James Bernauer et Jeremy R. Carrette (dir.), Michel Foucault and Theology. The Politics of Religious Experience, Londres, Ashgate, 2004.

4. Jonathan Tran, Foucault and Theology, New York, T\&T Clark International, 2011.

5. Ajoutons à cette liste rapide trois autres travaux consacrés de près ou de loin à une telle question: Bernauer James W., Michel Foucault's force of flight. Toward an Ethics for Thought, New Tork, Humanity Books, 1990; Jeremy R. Carrette (dir.), Religion and culture. Michel Foucault, London, Routledge, 1999; Galston David, Archives and the Event of God. The Impact of Michel Foucault on Philosophical Theology, Québec, McGill-Queen's University Press, 2011.
} 


\section{Labyrinthe, $n^{\circ} 40$}

optant en quelques sortes pour un " retour aux textes (ou cours) mêmes » contre la tradition d'interprétation, majoritairement anglo-saxonne sur la question, se contentant, en guise de justification, d'un simple renvoi au corpus foucaldien lui-même: « les références à l'héritage chrétien sont constantes dans l'œuvre de Michel Foucault ${ }^{6} \gg$. De l'introduction à l'Histoire de la folie aux derniers cours au Collège de France sur la parrêsia, en passant par l'ouverture de Naissance de la clinique, les articles plus « littéraires » des années 1960, sans oublier l'ensemble des occurrences dispersées dans bon nombre de cours des années 1970 tels que Les Anormaux ou Sécurité, territoire, population, il est vrai qu'il n'est pas difficile, pour le chercheur, de trouver sous la plume de Foucault des références au christianisme. Le problème n'est pas, alors, celui de faire dire davantage aux textes, mais plutôt d'arriver à connecter, agencer, organiser cette multiplication d'occurrences en une vision cohérente. Telle est la tension principale de l'entreprise: « la possibilité ou non de trouver une approche systématique du christianisme chez le philosophe français ${ }^{7}$ ». C'est la question première à laquelle Michel Foucault et le christianisme tente de répondre.

Pour qui est familier des textes foucaldiens déjà connus, et notamment de la recrudescence des discussions sur le christianisme dans les cours au Collège de France des années 1970, une première réponse vient à l'esprit : tant par le biais du projet d'histoire politique de la vérité (tel que nous le trouvons par exemple formulé dans les conférences données à l'Université pontificale catholique de Rio de Janeiro en $1973^{8}$ ) que dans le projet d'une histoire de la sexualité (telle que La Volonté de savoir le formule en $1976^{\circ}$ ), ce qui intéresse Foucault dans le christianisme se rapporte principalement aux techniques d'aveu qui s'y sont constituées. C'est en effet en étudiant l'évolution des dispositifs de pouvoir distincts des seules formes de la contrainte ou de l'interdiction (modèle légal ou juridique), ceux qu'il désigna par les notions de « discipline », " contrôle », puis " biopouvoir » ou " gouvernementalité », reposant sur une conduite de soi liée à un dire vrai sur soi, que Foucault entreprit de remonter généalogiquement au christianisme, perçu comme une des

\footnotetext{
6. Philippe Chevallier, op. cit., p. 11

7. Ibid. p. 12.

8. Michel Foucault, «La vérité et les formes juridiques ", in Dits et Ecrits, I, Gallimard, coll.

«Quarto", rééd. 2001, p. 1406-1514.

9. Michel Foucault, La Volonté de savoir. Histoire de la sexualité I, Gallimard, coll. « Tel », 1976.
} 


\section{Les Foucault(s) du christianisme}

sources principales de telles pratiques. L'aveu chrétien intéressa Foucault, selon une telle lecture, en cela qu'il fut l'une des premières techniques, et l'une des plus systématiques, permettant de lier le sujet à lui-même, à sa propre vérité, et de le rendre ainsi « sujet », à proprement parler, de ses désirs et de ses actes.

C'est un peu contre cette idée première qu'argumente Chevallier, en s'appuyant sur deux textes jusqu'alors inédits et qui viennent d'être publiés. D'abord la série de leçons données par Foucault en 1981 à l'Université catholique de Louvain sous le titre Mal faire, dire vrai. Fonction de l'aveu en justice ${ }^{10}$. Ensuite l'ensemble du cours au Collège de France de l'année 1979-1980, Du Gouvernement des vivants ${ }^{11}$, portant intégralement, mise à part son introduction, sur le christianisme naissant, et plus précisément sur la mise en place des pratiques de préparation au baptême ou à la deuxième pénitence entre les $\mathrm{II}^{\mathrm{e}}$ et $\mathrm{IV}^{\mathrm{e}}$ siècles. Si Chevallier accorde une place centrale à ces textes dans son travail, c'est qu'ils portent, selon lui, une lecture foucaldienne du christianisme assez différente de celle proposée dans Les Anormaux (1975) ou La Volonté de savoir (1976). Le commentateur révèle notamment une certaine autonomisation du domaine du savoir vis-à-vis du domaine du pouvoir, notamment par le développement, en 1980, d'un nouveau paradigme, celui de « régime de vérité ». L'autre élément mis au jour par ces cours, pour Chevallier, est une sorte de réouverture de questions ou d'enjeux déjà présents dans la série d'articles que Foucault consacra, dans les années 1960, à Blanchot, Bataille, Klossowski ou Flaubert et ne pouvant donc pas être directement rapportée aux réflexions autour du pouvoir et de l'aveu. Jeu de pontage donc; jeu de court-circuitage, diront certains. En opérant une telle liaison entre les années 1980, où reviennent les questions d'épistémologie et d'histoire des idées, et les années 1960, où la question politique n'est pas encore la préoccupation principale, c'est au fond une sorte de parenthèse sur le second pôle, celui des années 1970 - le premier qui vient à l'esprit, justement, celui des pratiques d'aveu - que jette Chevallier, ce qui lui permet d'appuyer

10. Michel Foucault, Mal faire, dire vrai. Fonction de l'aveu en justice. Cours de Louvain, 1981, Presses Universitaires de Louvain, 2012.

11. Michel Foucault, Du Gouvernement des vivants. Cours au Collège de France, 1979-1980, Seuil/ Gallimard, coll. « Hautes Etudes », 2012. 


\section{Labyrinthe, $n^{\circ} 40$}

l'une de ses thèses principales: « le christianisme pour Foucault n'est pas d'abord la religion de l'obéissance et de l'aveu ${ }^{12} »$.

Une telle entreprise propose une lecture nouvelle de Foucault, de l'objet « christianisme » dans ses recherches, mais aussi des points épistémologiques importants comme par exemple la notion de « régime de vérité » théorisée dans le cours de 1980 et jusqu'à aujourd'hui peu étudiée. On peut envisager l'apport de ce travail sur deux plans : celui de la méthode, et celui des thèses avancées par Michel Foucault et le christianisme.

L'intelligence du livre de Philippe Chevallier tient tout d'abord à son hypothèse méthodologique première: se donner un objet « christianisme ", en repérer les différents moments d'émergence dans le travail de Foucault, mais aussi constater à travers lui les approches toujours différentes que celui-ci adopte. Comparons avec les travaux anglo-saxons précédemment évoqués menés à propos de « Foucault et le christianisme ». À bien des égards, la posture adoptée par Chevallier en représente le négatif. En effet, dans ces recherches, deux questions structurent la quasi-totalité des approches. L'une se propose de repérer les « dimensions religieuses ${ }^{13}$ » évidentes, probables ou souterraines des postures, méthodes ou thèses foucaldiennes. L'autre se propose de puiser chez le philosophe français quelques outils pouvant être utiles aux réflexions théologiques contemporaines ${ }^{14}$. On le comprend, de tels axes de recherche, qu'il n'est pas ici question de discuter, ont nécessairement tendance à figer une « pensée Foucault». Ces postures réclament, comme matériau premier, un système philosophique et méthodologique relativement fixe et constant, matériau sur lequel et à partir duquel les deux types d'investigations ici schématisés peuvent se développer. Il en va tout autrement pour Philippe Chevallier. Là où ces approches ont besoin d'une sorte de « Foucault a priori » à partir de quoi penser a posteriori ses « dimensions religieuses » d'une part, ses conséquences ou ses applications théologiques d'autre part, Michel Foucault et le christianisme se donne en somme un «christianisme $a$ priori », c'est-à-dire un « objet christianisme » se retrouvant ici et là à travers l'œuvre foucaldienne, non pas lieu à partir duquel se dessinerait

12. Philippe Chevallier, op. cit., p. 15.

13. Jeremy R. Carrette, Foucault and Religion, op. cit., p. 1.

14. C'est le travail notamment mené par Jonathan Tran, op. cit. 


\section{Les Foucault(s) du christianisme}

un Foucault constant et répétitif, mais bien plutôt lieu à partir duquel les multiples déplacements composant l' "œuvre » foucaldienne apparaissent. Toute la subtilité de Chevallier tient ainsi à avoir su se glisser dans ce « christianisme » en tant qu'objet d'étude apparaissant régulièrement sous la plume et dans les paroles de Foucault pour pouvoir, à partir de là, étudier les évolutions du regard qui se pose et se repose sur lui. Le regard changeant de Foucault observé depuis cet objet « christianisme » : voilà en somme comment fonctionne Michel Foucault et le christianisme.

On pourrait s'attarder sur de nombreux aspects concernant le contenu de ce travail: l'analyse méticuleuse de la transformation de la méthode de Foucault, de sa manière de faire cours et surtout d'utiliser et de lire les textes anciens est un véritable apport ${ }^{15}$; la relecture des articles « littéraires » des années 1960 ou du débat avec Jacques Derrida sur le cas Descartes dans l'Histoire de la folie, à la lumière des problèmes traités dans le cours de $1980^{16}$ l'est tout autant. Mais nous nous concentrerons ici sur la thèse principale traversant l'ensemble de ces analyses, leur question la plus délicate aussi, c'est-à-dire la proposition de déconnexion ou de détachement - au moins relatifs - de l'approche foucaldienne du christianisme vis-à-vis de la problématique des techniques d'aveu. Incontestablement, l'écoute du cours de 1980, tout comme la lecture des diverses références au christianisme des écrits foucaldiens des années 1960, invitent à proposer une vision du christianisme plus complexe que celle présentée dans certains cours des années 1970. La difficulté consiste cependant tout particulièrement, et peut-être qu'ici Chevallier ne va pas assez loin, à pouvoir cibler ou exprimer de manière forte les fonctions ou intérêts « alternatifs » qu'une étude foucaldienne du christianisme pourrait alors comporter. Pour le tenter, il explore principalement deux voies.

La première s'appuie sur les déplacements qu'impliquent, pour Foucault, le fait de penser le christianisme comme une forme de « gouvernementalité » d'une part, une forme de « régime de vérité » d'autre part. Il est vrai qu'en 1980 cette dernière notion acquiert une importance toute particulière et tend même à remplacer, selon les propres mots de Foucault, les cadres d'analyses, qu'il juge alors dépassés, de son ancienne méthode

15. Cf. Philippe Chevallier, op. cit., p. $155 \mathrm{~s}$

16. Cf. Ibid., p. 172s. 


\section{Labyrinthe, $n^{\circ} 40$}

d'approche des relations entre pouvoir, savoir et subjectivité. Tentant de préciser un tel déplacement, Chevallier le synthétise ainsi :

À l'affirmation massive de 1976 que la vérité n'est jamais hors pouvoir, fait place un premier partage entre la normativité interne du discours scientifique et les obligations que les sujets ont à son égard. Pour reprendre les termes mêmes de Foucault, il s'agit, en science, de distinguer ce qui permet de dire «c'est vrai » de ce qui oblige à dire « donc, je m'incline ». Ce « donc », à l'intérieur duquel un sujet s'engage, n'est pas une conséquence de la vérité de la proposition; il est « historique et culturel ». [...] Mais dire cela, c'est en même temps rendre à la cohérence interne d'un discours scientifique son autonomie et son efficacité propre, irréductible aux mécanismes sociaux de circulation et de régulation des discours ${ }^{17}$.

Une telle remarque remet en question l'une des positions importantes des études foucaldiennes des années 1970 : l'intrication essentielle entre « savoir » et « pouvoir », y compris dans le cas du savoir « scientifique ». Ici comme ailleurs, c'est un certain retour aux thèses des années 1960 que laisse percevoir l'auteur. Il nous semble cependant, mais d'autres pages devront préciser ce point, qu'une telle lecture des « régimes de vérité » allant dans le sens d'une certaine « autonomisation » de la science est sujette à discussion; mieux : que le cours de 1980 se donnant en partie pour tâche de développer cette notion reste bien souvent dans le flou, et surtout propose, au fil des multiples retours de Foucault sur le sujet, des analyses parfois divergentes. L'exemple des deux logiciens tel qu'il apparait dans la cinquième leçon à laquelle fait référence Chevallier ici en est un bon exemple. Foucault y prend l'exemple de deux logiciens initialement en désaccord, dont l'un déclare pourtant, à la suite de la démonstration de l'autre: «c'est vrai, donc je m'incline ». Dans cet exemple, comme le rapporte Chevallier, le « c'est vrai » est d'ordre strictement logique, alors que le « donc » invitant le logicien à s'incliner dans le cas d'une démonstration logique incontestable ne peut pas être du même ordre et relève ainsi de ce que nous pourrions nommer la déontologie du logicien, renvoyant ainsi à ce que Foucault nomme un « régime de vérité ». À l'inverse de la conclusion qu'en tire Chevallier, voyant dans cet exemple la

17. Ibid., p. 94 


\section{Les Foucault(s) du christianisme}

distinction opérée par Foucault entre un niveau relevant uniquement des règles logiques et scientifiques et un autre relevant dudit régime et donc de considérations extra-logiques, l'écoute de la leçon dans sa globalité pourrait autoriser une interprétation inverse. Cet exemple fut en effet pris, dans la chronologie du cours, à la suite d'une objection formulée contre la notion même de " régime de vérité » et consistant à dire que s'il y a « régime », autrement dit s'il y a contrainte ou règle extérieures, alors il n'est pas question du « vrai ». L'hypothèse que sous-tend une telle objection serait alors que le vrai n'aurait besoin de rien d'autre que de lui-même pour « contraindre » ou « incliner », que le vrai ne serait donc pas seulement « index sui» mais également « rex sui, lex sui, judex $s u i »$. Face à une telle position, Foucault voulait alors avancer sa propre thèse selon laquelle « il n'est pas vrai que la vérité ne contraigne que par le vrai ». C'est à cette occasion qu'il prit alors l'exemple des deux logiciens, qu'il est donc possible de concevoir comme un argument $a$ fortiori. Il s'agissait alors de montrer que même dans la logique, cœur de la science moderne, un « régime de vérité » s'exerce, qu'il consiste à lier le « je m'incline » du logicien au « c'est vrai » logique, et ainsi que dans la logique comme ailleurs, il n'est pas possible d'étudier le savoir et son fonctionnement en ayant exclusivement recours à ses propres règles internes d'argumentation logique.

Mais c'est surtout la deuxième voie empruntée par Philippe Chevallier que nous voudrions discuter. Elle trouve ses racines dans des réflexions d'ordre lexicographique. L'auteur remarque tout d'abord, à juste titre, un changement dans la manière qu'a Foucault, en 1980, de présenter et qualifier l'enjeu généalogique présidant à l'étude du christianisme. Il ne s'agit plus, alors, de proposer une généalogie des pratiques d'aveu mais bien plutôt de la «subjectivité occidentale ». Nous pourrions cependant nous demander si ces deux objets ne sont pas, d'une manière ou d'une autre, intimement reliés. En effet, si Foucault définit la subjectivité comme « le rapport de soi à soi, l'exercice de soi sur soi ${ }^{18}$ », quelque chose lui semble être tout particulièrement spécifique à la subjectivité occidentale qu'il veut étudier : « un rapport au discours et un rapport à l'aveu qui est absolument caractéristique de notre civilisation ${ }^{19}$ ». C'est donc la généalogie de ce rapport entre subjectivité et vérité, autrement

18. Michel Foucault, Du Gouvernement des vivants, op. cit., p. 124

19. Ibid., p. 206. 


\section{Labyrinthe, $n^{\circ} 40$}

dit de cette forme spécifique de rapport à soi appuyée sur une vérité que le sujet peut trouver « sur soi » et « au fond de soi », qu'entame Foucault en 1980. Cette caractéristique, il l'avait repérée à propos de dispositifs propres à l'époque moderne et contemporaine; il avait proposé d'en identifier l'antécédent généalogique dans le pastorat chrétien et l'organisation « aboutie » et «mûre » de l'aveu à la fin du Moyen Âge; il se donne pour tâche, en 1980, d'en établir les conditions d'émergence dans les trois premiers siècles chrétiens.

Une telle « objection » que nous pourrions adresser à Chevallier n'est pourtant pas tout à fait satisfaisante. Elle ne rend par exemple pas compte d'un second déplacement d'ordre lexicographique relevé par lui, concernant la définition du « christianisme » lui-même, de la « religion de l'aveu » des années 1970 devenant, en 1980, la « religion du salut dans l'imperfection ${ }^{20} »$. Ici encore, il serait facile de relier une telle définition à la première, la situation toujours problématique et jamais aboutie d'un salut toujours déjà et toujours encore coloré d'imperfection rendant non seulement nécessaire mais également répétable à l'infini les pratiques d'aveu. Ici également, pourtant, il faut se méfier des réponses ou objections trop rapides. La lecture du cours de 1980 montre bien, en effet, qu'une telle situation n'a pas donné naissance, en tout cas durant les siècles qu'étudie alors Foucault, à cette pratique particulière de l'aveu qui n'apparaîtra que bien plus tard. Plus fondamentalement ensuite, c'est une autre question qu'invite à poser Michel Foucault et le christianisme: une telle définition du christianisme comme la religion du salut dans l'imperfection, qui ne manque certes pas de pertinence pour toute pensée, historique et philosophique, du christianisme, peut-elle être détachée, dans la trajectoire de Foucault, du projet de généalogie de la subjectivité occidentale et de l'aveu? Si nous avons vu qu'une réponse négative pouvait être spontanément apportée à une telle question, c'est une reformulation plus positive que le travail de Chevallier invite à proposer: le salut chrétien et sa dimension problématique seraient-ils susceptibles, en eux-mêmes, de pouvoir attirer l'intérêt foucaldien indépendamment de la place qu'ils pourraient avoir dans une telle généalogie ?

Devant une réponse trop catégorique rattachant systématiquement de telles études au projet généalogique d'histoire de la sexualité que nous avons évoqué, il faudrait par exemple rappeler qu'ici comme peut-être pour

20. Philippe Chevallier, op. cit., p. 346. 


\section{Les Foucault(s) du christianisme}

l'ensemble du « dernier Foucault », le commentateur se trouve toujours dans une situation bien inconfortable, pris entre deux mouvements: celui ayant conduit Foucault à cette étude et qui relève effectivement de son projet d'histoire de la sexualité et de la subjectivité occidentale, et celui pouvant potentiellement résulter ou naître en chemin ou à partir de celui-ci, mouvement que la courte période séparant le cours de 1980 de la mort de Foucault n'a malheureusement pas permis de bien percevoir et oblige à devoir seulement conjecturer. C'est vers de telles conjectures, croyons-nous, qu'invite à avancer Philippe Chevallier et que nous proposerions, pour notre part, d'envisager plus avant encore qu'il ne le fait; en relevant l'intérêt évident et manifeste qu'a exercé sur Foucault tout un ensemble de questions que pourrait recouvrir la grande catégorie de la " spiritualité », intérêt dont on pourrait dessiner l'évolution à partir de la « spiritualité politique » émergeant dans l'analyse du Soulèvement iranien de 1978 jusqu'aux pratiques parrèsiastiques des derniers cours au Collège de France en passant par le « souci de soi » du cours de 1982 et à l'intérieur de laquelle la question du « salut dans l'imperfection » du christianisme étudié en 1980 aurait toute sa place. Nous pourrions ainsi proposer un tout autre partage des différentes « périodes » des analyses foucaldiennes, fondé non plus uniquement sur les champs historiques par lui étudiés, apporter des clefs de compréhension nouvelles quant à sa lecture des textes antiques, mais aussi tenter de délimiter une série de problèmes et d'enjeux nouveaux se dessinant alors à partir mais aussi en se détachant progressivement de ce qu'il serait alors possible de nommer, avec un brin de provocation, la « prison » du projet d'histoire de la sexualité dans les six à huit dernières années de travail de Foucault. Il nous semble en tout cas, par-delà la multitude de questions et de discussions que provoque bien heureusement le livre de Chevallier, que se trouve là l'une des questions essentielles qu'il suscite in fine, question dont il serait bien triste de sous-estimer l'intérêt. 